\title{
The value of interleukin 6 as a peripheral diagnostic marker in schizophrenia
}

\author{
Kayla A. Chase ${ }^{1,2}$, Jackson J. Cone ${ }^{3}$, Cherise Rosen ${ }^{1}$ and Rajiv P. Sharma ${ }^{1,4^{*}}$
}

\begin{abstract}
Background: Associations between a pro-inflammatory state and schizophrenia have been one of the more enduring findings of psychiatry, with various lines of evidence suggesting a compelling role for IL-6 in the underlying pathogenesis of schizophrenia.

Methods: In this study, we examined IL-6 mRNA levels by real-time RT-PCR from fresh extracted peripheral blood mononuclear cells (PBMC) in normal controls and participants with schizophrenia.

Results: We found that peripheral PBMC IL-6 mRNA levels, in the absence of any other information, reliably discriminated between a diagnosis of schizophrenia and normal controls. Furthermore, in participants with schizophrenia, we also found elevated levels of IL-6 mRNA with earlier ages of illness onset and worse positive symptom presentation, as measured by the Positive and Negative Syndrome Scale.

Conclusions: These findings provide important and continued support for a pathophysiological role of inflammation in patients with schizophrenia. Future utilization of peripheral IL-6 mRNA levels could be clinically useful during an initial diagnosis and help tailor individualized treatment plans for patients with schizophrenia.
\end{abstract}

Keywords: Schizophrenia, Lymphocyte, Interleukin-6, Biomarker, PBMC

\section{Background}

Patients with schizophrenia have altered immune function [1]. This, coupled with studies indicating the impact of maternal illness on offspring development [2, 3], strongly suggests a link between the inflammasome and schizophrenia. As such, interleukin 6 (IL-6), which can function as both a pro- and anti-inflammatory cytokinehas been examined for its role in the development, course, symptomology, and applicability as a biomarker of schizophrenia.

Various lines of evidence suggest a compelling role for IL-6 in the underlying pathogenesis of schizophrenia. First and foremost, IL-6 and sIL-6R serum levels are significantly elevated in patients with schizophrenia $[4,5]$, with implications for symptom severity [6]. The singlenucleotide polymorphism (SNP) G/C at position -174 of the IL-6 gene may also result in increased susceptibility

\footnotetext{
* Correspondence: rsharma@psych.uic.edu

${ }^{1}$ The Psychiatric Institute, University of Illinois at Chicago, 1601 W. Taylor St., Chicago, IL 60612, USA

${ }^{4}$ Jesse Brown Veterans Affairs Medical Center, 820 South Damen Avenue (M/ C 151), Chicago, IL 60612, USA

Full list of author information is available at the end of the article
}

to schizophrenia, although this seems to be highly dependent on environmental factors [7]. This SNP has also been linked to overall symptomology in schizophrenia $[8,9]$. Maternal immune activation models of neurodevelopmental insults have heralded insight into the role of IL-6 and schizophrenia. Maternally produced cytokines can both cross the placenta [10] and induce an acute-phase reaction in response to immune challenges [11]. A single maternal dose of IL-6 results in schizophrenic-like behavioral changes in rodent offspring [12]. Furthermore, inhibiting IL-6 action in response to an immune challenge can normalize these behaviors in adult offspring [12]. Thus, multiple lines of evidence link inflammatory factors, specifically IL-6, with mental illness and related symptomology.

In this study, we demonstrate that IL- 6 mRNA levels from freshly extracted peripheral blood mononuclear cells (PBMC) could be a useful and easily clinically accessible biomarker for a diagnosis of schizophrenia. There is a general lack of correlation between cytokine serum levels and PBMC gene expression [13]. As such, measurement of mRNA in PBMC is tissue specific and 
avoids interpretation regarding the source of serum levels of cytokines, which could emanate from the liver, muscle, adipose and other tissues. Additionally, we attempted to elucidate the role of IL-6 in disease onset and symptom presentation in schizophrenia.

\section{Methods}

\section{Participants}

The sample included 106 participants, including 53 patients with schizophrenia and 53 healthy individuals recruited from the University of Illinois at Chicago (UIC) medical center and surrounding community. All study procedures were approved by The Office for the Protection of Research Subjects at UIC. All participants provided written informed consent before participating in any study procedures. Patients with schizophrenia were assessed by experienced diagnosticians (MD or $\mathrm{PhD}$ ) via Structured Clinical Interview for DSM Disorders (SCID) interview, DSM-IV-TR criteria and all available information. Clinicians established a consensus before final assignment to diagnostic group. All participants were between 21-65 years of age and in good physical health (with no reported infections or autoimmune diseases), and exclusion criteria were: a history of neurological disease or head trauma, lifetime history of substance/alcohol dependence or recent substance abuse, and pregnancy for women. Exclusionary criteria for control participants also included a major Axis I disorder (assessed by a SCID interview) and a known first-degree familial history of psychosis. Participant demographics are listed on Table 1.

At the time of sampling, $55 \%(n=29)$ of the patients with schizophrenia were evaluated while hospitalized on the inpatient psychiatric unit and $45 \%(n=24)$ were evaluated in the psychiatric outpatient clinic. Prescribed antipsychotic medication was coded on all participants as follows: Haloperidol $=2$, Fluphenazine $=2$, Perphenazine $=2, \quad$ Risperidone $=19, \quad$ Olanzapine $=3$, Quetiapine $=7, \quad$ Ziprasidone $=3, \quad$ Aripiprazole $=4$, Lurasidone $=3$, Iloperidone $=1$, Paliperidone $=2$, (a total of $n=48$ ). Five patients were unmedicated at the time of the blood draw. Due to this heterogeneity, all antipsychotic use was converted to Chlorpromazine (CPZ) units $[14,15]$.

\section{Clinical measures}

From all patients with schizophrenia, age of symptom onset was collected. Additionally, the Positive and Negative Syndrome Scale (PANSS) was administered to all clinical participants. The PANSS is used to generate measures of positive, negative, and general psychopathology through using a 7-point scale, with ratings ranging from 1 (symptom is absent) to 7 (symptom is extreme) [16].
Table 1 Means and standard deviations of participant demographics comparing healthy controls and participants with schizophrenia in PBMC samples

\begin{tabular}{lll}
\hline Demographic & Healthy Controls & $\begin{array}{l}\text { Patients with } \\
\text { Schizophrenia }\end{array}$ \\
\hline Sex (M/F) & $24 / 29$ & $34 / 19$ \\
& $\mathrm{t}=1.97 ; p=0.052$ & \\
& $35.5 \pm 11.4$ & $36.8 \pm 11.8$ \\
Age (Mean \pm SD) & $\mathrm{t}=-0.57 ; p=0.57$ & \\
Race (\%) & $17(32)$ & $4(8)$ \\
Caucasian, non-Hispanic & $18(34)$ & $39(73)$ \\
Black, non-Hispanic & $11(21)$ & $3(6)$ \\
Asian or other Pacific Islander & $7(13)$ & $7(13)$ \\
Hispanic & $\mathrm{F}(1,105)=0.10 ; p=0.75$ \\
BMl (Mean \pm SD) & $29.3 \pm 8.5$ & $31.6 \pm 7.7$ \\
& $\mathrm{t}=-1.36 ; p=0.18$ & \\
Waist Circumference & $39.8 \pm 8.45$ & $42.0 \pm 8.17$ \\
& $\mathrm{t}=-1.26 ; p=0.209$ &
\end{tabular}

Statistical differences between the various parameters were determined by a Student's $t$-test or one way ANOVA, with results indicated

\section{Peripheral blood mononuclear cell extraction}

A blood sample was obtained by sterile venipuncture into a $0.5 \mathrm{M}$ EDTA anticoagulant filled tube. Peripheral blood mononuclear cells (PBMC) were extracted utilizing the Ficoll-Paque ${ }^{\circ}$ method (GE Healthcare Life Sciences) [17]. Subsequent washing of the cream-colored interlayer was performed using the Hanks Balanced Salt Solution (HBSS - Gibco \#14170-161) to remove any remaining platelets, plasma or other contaminants. PBMC samples were pelleted at 2,000RPM for ten minutes at $10{ }^{\circ} \mathrm{C}$ and frozen in TRIzol reagent $-80{ }^{\circ} \mathrm{C}$ until mRNA extraction.

\section{mRNA extraction and quantitative real-time RT-PCR}

Total RNA was isolated using TRIzol reagent (Life Technologies) and treated with DNase (Ambion/Life Technologies AM1906) after extraction. Only total RNA extracts with an OD260/OD280 ratio above 1.96, indicating relatively pure RNA, were processed for RT-PCR, the remainder undergoing re-extraction using the standard phenol-chloroform extraction protocol. Total RNA was used to prepare cDNA via the Applied Biosystems High Capacity cDNA Reverse Transcription Kit (\#4368814). For detection and measurement of expression, Fermentas Maxima SYBR Green/ROX qPCR Master Mix (\#K0222) was used. PCR mixtures were run on a Thermo Scientific PikoReal real-time PCR System using the following conditions: $95{ }^{\circ} \mathrm{C}$ for $10 \mathrm{~min}$, followed by 40 cycles of $95{ }^{\circ} \mathrm{C}$ for $30 \mathrm{~s}, 60{ }^{\circ} \mathrm{C}$ for one minute and $72{ }^{\circ} \mathrm{C}$ for one minute. Cycle threshold (CT) value was 
used for relative quantification, and all values were normalized to three housekeeping genes, GAPDH, TFRC and $\beta$-Actin (chosen for their previously, unpublished determined stability across diagnostic groups) using a geometric mean, and run in triplicate [18-20]. Primer sequences are listed in Table 2.

\section{Statistical analysis}

SPSS (version 15.0 for Windows) was used for all statistical analyses. Analysis of all mRNA data was conducted on CT levels of the gene of interest, normalized to the geometric mean of three control genes. Data is presented as mean values \pm standard error. A probability level of $p<0.05$ was the criterion to achieve statistical significance.

\section{Results}

\section{Shapiro Wilk analysis for normality}

Normality of IL-6 mRNA distributions in both diagnostic groups was assessed by Shapiro-Wilk W tests where a $p<0.05$ rejects the null hypothesis that the distribution is normal. Results indicate that the distribution for both controls and participants with schizophrenia was not normal, $p=0.001$ and $p=0.008$ respectively. Thus, for all further data analysis we utilized non-parametric tests, including the Mann-Whitney U, Spearman correlations, two-sample Kolmogorov-Smirnov test and the Receiver Operating Characteristic.

\section{Comparison of IL-6 mRNA expression levels in PBMC}

We performed a Mann-Whitney U analysis with PBMC IL-6 mRNA as the dependent variable and diagnosis as the independent variable. We discovered that participants with a diagnosis of schizophrenia had significantly higher IL-6 mRNA levels when compared to normal controls $(U=632.5, p<0.001)$. There were no significant differences between diagnosis and measurements of sex, age, race, BMI or waist circumference, as determined by either a Student's $t$-test or a One Way Analysis of Variance (ANOVA; Table 1). The mean value of IL-6 mRNA levels for control participants is $3.11 \pm 1.7$ (standard deviation). The mean value for PBMC IL-6 mRNA levels in participants with schizophrenia is $5.64 \pm 3.0$ (Fig. 1).

Table 2 Real-time RT-PCR mRNA primers utilized for this study

\begin{tabular}{lll}
\hline $\begin{array}{l}\text { Primer } \\
\text { Name }\end{array}$ & Left Primer & Right Primer \\
mRNA Expression Primers & \\
\hline IL-6 & AAAGAGGCACTGGCAGAAAA & AGCTCTGGCTTGTTCCTCAC \\
GAPDH & CGAGATCCCTCCAAAATCAA & TTCACACCCATGACGAACAT \\
TFRC & CACCAACCGATCCAAAGTCT & AAAATCCGGTGTAGGCACAG \\
BActin & TGAAGGTAGTTTCGTGATGC & TCCCTGGAGAAGAGCTACGA \\
\hline
\end{tabular}

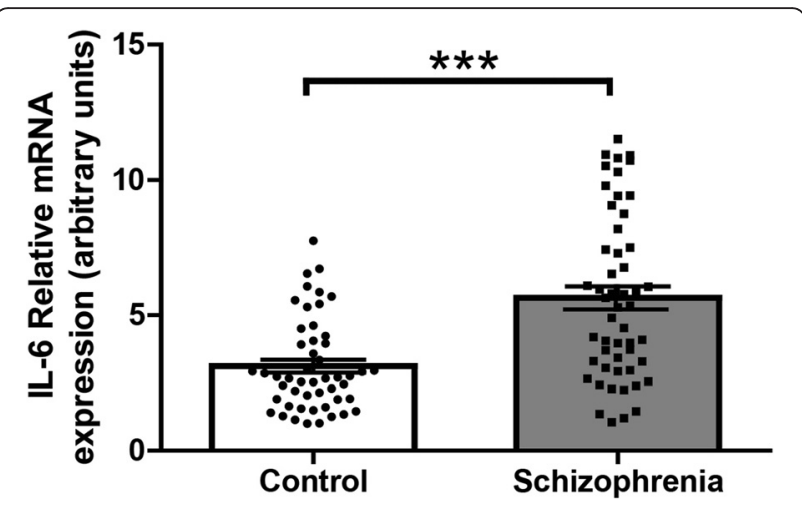

Fig. $1 \mathrm{lL}-6 \mathrm{mRNA}$ levels in peripheral blood mononuclear cells (PBMC). IL-6 mRNA levels were significantly increased in participants with schizophrenia when compared to normal controls. Data presented as mean and SEM, $p<0.05$

\section{IL-6 mRNA correlates with clinical scales}

We found that higher PBMC IL-6 mRNA levels were associated with earlier onset of psychiatric symptoms (PBMC IL-6 mRNA x age of symptom onset; Spearman's $\rho=-0.23, p=0.02$; Fig. 2a). We also found that increases in IL-6 mRNA levels were correlated with increased presentation of positive symptoms, as measured by the PANSS (Spearman's $\rho=0.35, p=0.014$; Fig. 2b).

\section{Two-sample Kolmogorov-Smirnov test}

A two-sample Kolmogorov-Smirnov (KS) test was used to compare the distributions of IL-6 mRNA levels between controls and participants with schizophrenia [21]. The KS test was significant $(p<0.001)$, supporting the hypothesis that the two samples originate from different distributions (Fig. 3).

\section{Receiver operating characteristic analysis}

A receiver operating characteristics (ROC) curve was generated to examine the diagnostic value of using IL-6 mRNA as a binary classifier (patient with schizophrenia vs. controls). For this analysis, the area under the ROC curve (AUC) represents the predictive value of using only PBMC IL-6 mRNA levels to accurately differentiate between participants with schizophrenia and healthy controls across all of IL-6 mRNA levels found in our sample. While an AUC of 0.50 indicates there is no diagnostic value of IL- 6 mRNA, an AUC greater than 0.50 indicates a greater than chance probability of successfully discriminating diagnoses from a randomly chosen sample of the IL- 6 mRNA data. Notably, the AUC of IL6 from PBMC was 0.76 , with a $95 \%$ confidence interval of $0.66-0.85$ ( $p<0.001$; Fig. 4). This result demonstrates that IL-6 mRNA levels can reliably discriminate between a diagnosis of schizophrenia from normal controls in our sample ( $25 \%$ better than chance). 

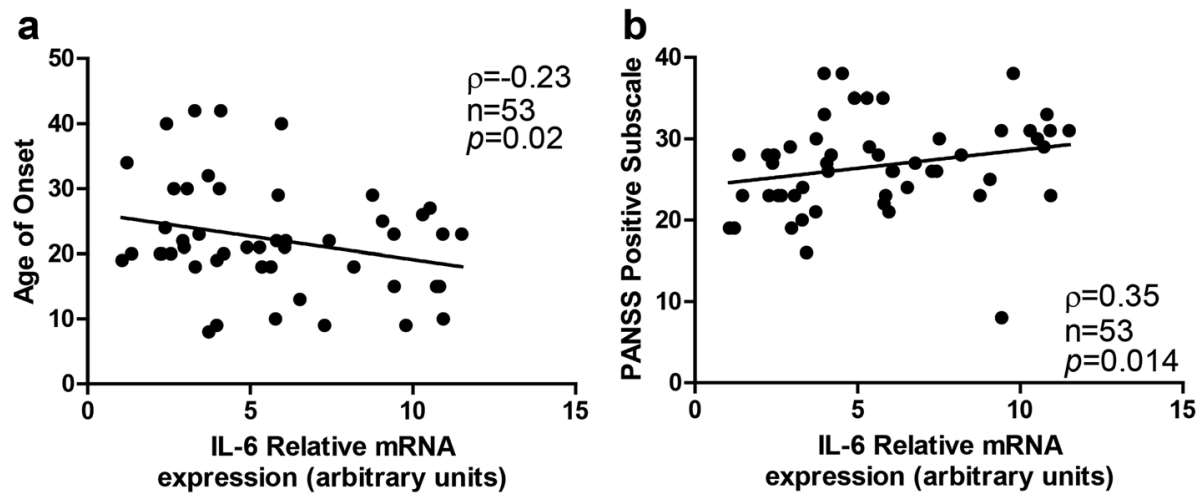

Fig. 2 Clinical correlates with IL-6 mRNA levels (a) IL-6 mRNA levels were increased in participants with an earlier age of illness onset. b IL-6 mRNA levels were higher in participants with increased positive symptom presentation, as measured by the Positive and Negative Syndrome Scale (PANSS). Spearman rho values, sample size and significance levels are all listed on individual graphs

\section{Discussion}

Our results demonstrate that IL-6 mRNA levels from freshly extracted PBMC are significantly upregulated in participants with schizophrenia when compared to healthy controls, thereby confirming our hypothesis. We also demonstrate that IL-6 mRNA levels are significantly elevated in patients who had an earlier age of onset, as well as those who demonstrated worse positive symptomatology. Finally, IL-6 levels may be a useful biomarker for a diagnosis of schizophrenia due to the predictive capabilities we demonstrate here.

Associations between a pro-inflammatory state and schizophrenia have been one of the more enduring findings of the field [4]. Our finding of an elevation in levels

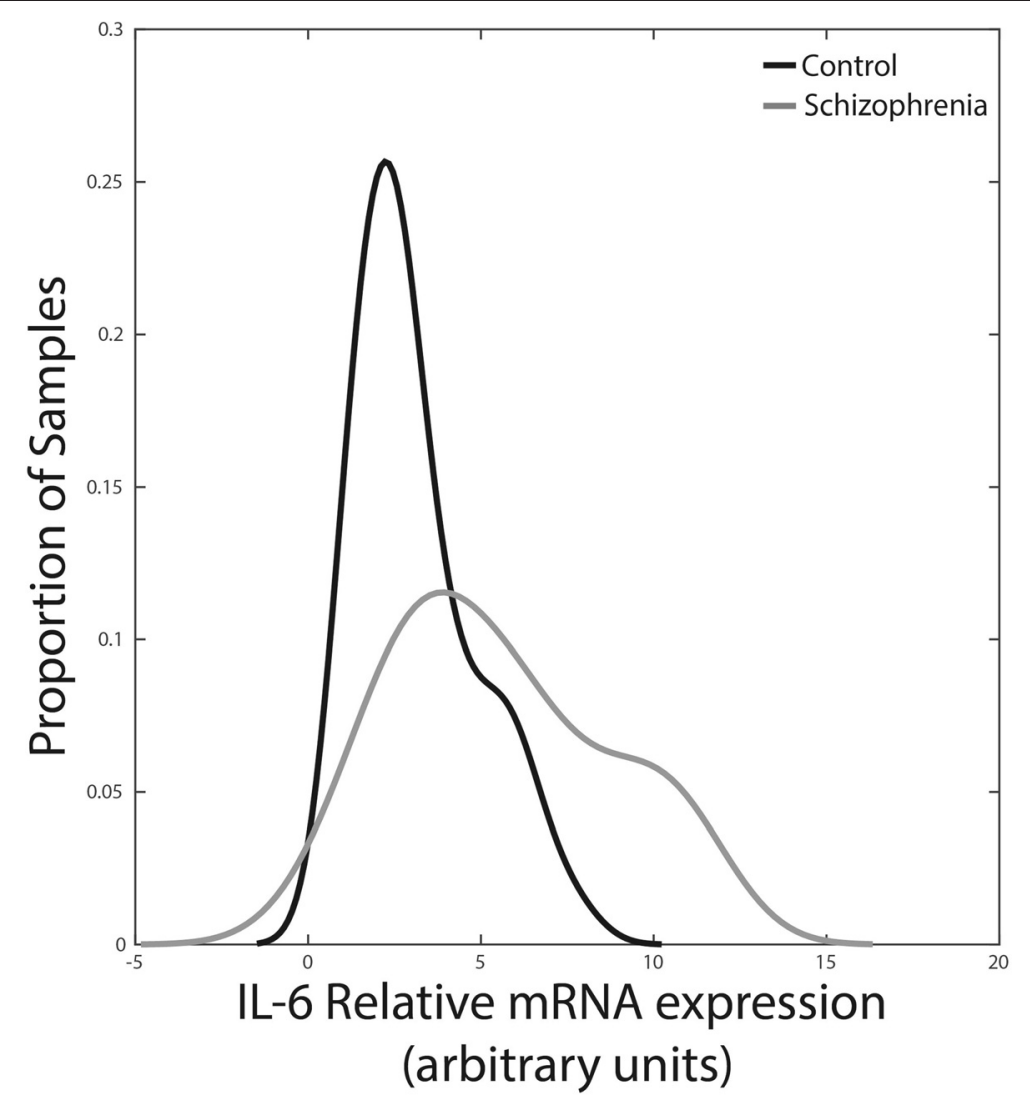

Fig. 3 Distributions of IL-6 mRNA levels between controls and participants with schizophrenia. The two-sample Kolmogorov-Smirnov test is significant $(p<0.001)$, demonstrating that the two diagnostic groups originate from different distributions 


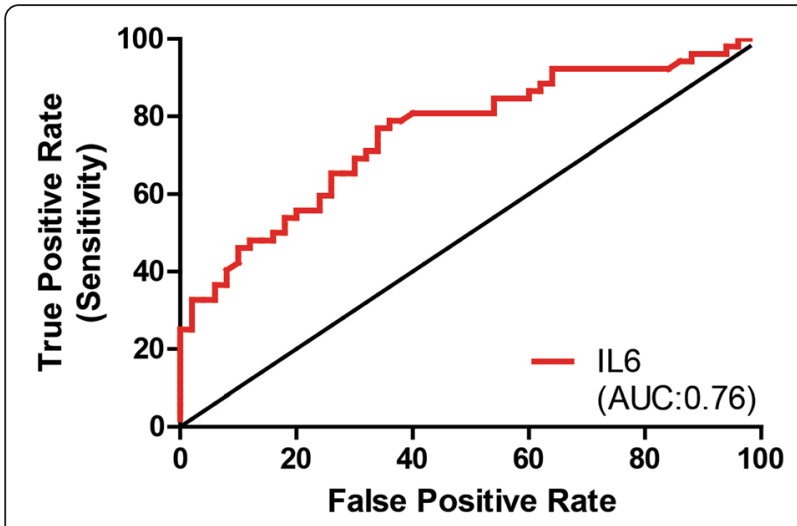

Fig. 4 A receiver operating characteristics (ROC) curve analysis of the diagnostic value of IL-6 mRNA from primary blood mononuclear cells (PBMC). An area under the curve (AUC) value of 0.76 indicates the predictive capability of IL-6 mRNA levels for a diagnosis of schizophrenia in our sample

of IL- 6 is consistent with the majority of the literature $[6,9,22,23]$. One such study, by Frydecka, et al., [23] found that serum IL-6 levels were significantly higher in participants with schizophrenia when compared to healthy controls. Additionally, in their sample, elevated IL-6 serum levels were associated with longer duration of illness. Our study complements and extends these findings. Importantly, IL-6 levels examined in this manuscript are mRNA from peripheral blood mononuclear cells. IL-6 levels measured in the serum, as in the Frydecka paper, can originate from multiple tissues, including adipose, muscle and liver, with particularly confounding effects given the well known metabolic disturbances in the schizophrenia population [24, 25]. Thus the demonstrated elevated serum IL-6 levels could have originated at any number of locations, thereby making the true source, cause, and implications difficult to interpret. Additionally, the Frydecka et al., study found no associations between symptom presentation, as measured by the PANSS, and serum IL- 6 levels, while we found a correlation with IL-6 mRNA levels and positive symptoms [23]. These differences may be attributable to the differences in type of biochemical sample examined between our two studies, paving the way for further discussion as to the importance of the location of the IL-6 signal and its role in mental illness.

Due to the heterogeneity of prescribed psychotropic medication in our naturalistic study, all antipsychotic use was converted to Chlorpromazine (CPZ) units. The impact of psychotropic medication on the inflammasome in participants with schizophrenia is a dynamic and novel field. Levels of proinflammatory cytokines are significantly elevated in female patients treated with clozapine, when compared to healthy controls [26]. However, these participants had significantly increased BMI levels when compared to normal controls, a phenotype associated with elevated cytokine levels, further complicating the interpretation. Additionally, treatment with olanzapine for 8 weeks reduces plasma IL-2 levels, but results in no changes in IL-6 or TNFa levels [27]. Treatment with chlorpromazine inhibits TNFa, but upregulates peripheral IL-10 levels in mice [28]. These findings all indicate that more research is needed regarding the uncontrollable variable of psychotropic medications play in the larger picture of inflammation and schizophrenia.

The association between IL-6 mRNA levels with age of onset and positive symptomatology is consistent with previous literature. In schizophrenia, serum IL-6 levels are positively correlated with PANSS Scores [29], an association our data replicates and extends on by examining this correlation with PBMCs. In schizophrenia, age of illness onset is a crucial determinant of clinical outcomes $[30,31]$, with a younger onset-age correlated with a worse disease prognosis and increased symptom presentation [32-34]. IL-6 levels are associated with younger psychosis onset, longer duration of illness and worse illness presentation [23], all indicators of worse disease course. IL- 6 has been proposed to act as a "state marker" for schizophrenia, with elevated serum levels seen in first episode psychosis and acutely relapsed patients [35]. These listed studies are all in line with our findings that the underlying pathophysiology of schizophrenia is characterized by a low-grade inflammatory state. It is important to the field to present multiple lines of consistent data regarding elevated levels of IL-6 in participants with schizophrenia in independent cohorts. Additionally, further studies need to be conducted examining IL-6 mRNA levels in participants with schizophrenia when compared to other psychiatric disorders, specifically depression.

Previous work has explored the use of IL-6 as a clinical predictor, but for infection in febrile neutropenia [36], not schizophrenia. In schizophrenia research, the receiver operating characteristic analysis has been used to examine the predictive value of specific microRNAs not associated with the immune system [37, 38]. Our work merges these two concepts to examine the predictive value of PBMC IL-6 mRNA levels in schizophrenia. Interestingly, evidence indicates that IL-6 levels are increased in schizophrenia-risk subjects, with highest levels demonstrated in those who transitioned from risk to acute psychosis [39]. This suggests that IL-6 could serve as a pre-diagnostic tool for identifying individuals who are more likely to suffer from the disease. In the clinic, an especially useful aspect of the ROC is criterion; a cutoff that attempts to maximize the likelihood of a true positive (accurate diagnosis) while minimizing the chance of an incorrect diagnosis (false positive). In our data set, using a cutoff IL-6 mRNA value of 4.9 
(arbitrary units) would result in a true positive rate of $53 \%$, while a false positive would occur $17 \%$ of the time. If the cutoff value is increased, the chance of a true positive increases, while false positives decrease. For example, a cutoff of 6.0 in our sample would be five times more likely to make a correct diagnosis of schizophrenia based solely on IL-6 mRNA levels in PBMC. In the clinic, physicians would have access to much more patient data, and thus could adjust their criterion as needed to ensure an accurate diagnoses.

\section{Conclusions}

Accessible biological markers are desperately needed to improve the timing and accuracy of a diagnosis of schizophrenia. These findings provide important support for a pathophysiological role for inflammation in patients with schizophrenia. While not strong enough to stand as a predictor of schizophrenia on its own, IL-6 mRNA levels may be yet another tool for clinicians to use during either an initial diagnosis or for tailoring individualized treatment plans for patients with schizophrenia. The application of accurate in vitro diagnostic tools that can accurately classify psychiatric disorders or identify disease subtypes could help to reduce illness duration and cost, including hospital services and loss of work, and improve treatment outcomes. Although such personalized medicine is still expensive, with the lightening speed of new technological development, these costs are rapidly decreasing [40]. In conclusion, although further studies using a larger cohort of participants are needed, data from the present study suggest that peripheral blood mononuclear cell level of IL-6 mRNA has diagnostic relevancy and predictive value in schizophrenia.

\section{Abbreviations}

PBMC: peripheral blood mononuclear cells; PANSS: Positive and Negative Syndrome Scale; AUC: area under the curve; ROC: receiver operating characteristics; IL-6: interleukin 6: SNP: single-nucleotide polymorphism; SCID: Structured Clinical Interview for DSM Disorders; HBSS: Hanks Balanced Salt Solution; CT: Cycle threshold; CPZ: Chlorpromazine; KS: two-sample Kolmogorov-Smirnov test.

\section{Acknowledgements}

The authors would like to thank all the subjects who participated in this study.

\section{Funding}

This work was supported in part by PHS grant (NIH) R01MH094358 (RPS).

\section{Availability of data and materials}

Given the sensitive nature of the data collected on each participant, the data used in this manuscript will not be made available to the public in order to protect participant identity, as full anonymity cannot be guaranteed. Potential collaborators are encouraged to contact the corresponding author directly.

\section{Authors' contributions}

KAC, JJC and RPS conceived the study and participated in its design and coordination. CR and RPS recruited participants and conducted all clinical measures. KAC carried out all biochemical methods. JJC and KAC performed the analysis. All authors read and approved the final manuscript.

\section{Authors' information}

Not applicable

\section{Competing interests}

The authors declare that they have no competing interests.

\section{Consent for publication}

Institutionally approved consent forms signed by all participants included sections regarding the use of deidentified data in manuscripts.

\section{Ethics approval and consent to participate}

All study procedures were approved by The Office for the Protection of Research Subjects at The University of Illinois at Chicago (UIC). UIC has three review boards for research involving human subjects, each of which reviews protocols according to their expertise. Study procedures conducted in this manuscript were approved by approval board IRB1 - "Biomedical and Biological Science Research". All participants provided written informed consent before participating in any study procedures.

\section{Author details}

${ }^{1}$ The Psychiatric Institute, University of Illinois at Chicago, 1601 W. Taylor St., Chicago, IL 60612, USA. Department of Psychiatry, The University of California, 9500 Gilman Drive, La Jolla, San Diego, CA 92093, USA ${ }^{3}$ Department of Neurobiology, The University of Chicago, 5812 S. Ellis, Chicago, IL 60637, USA. ${ }^{4}$ Jesse Brown Veterans Affairs Medical Center, 820 South Damen Avenue (M/C 151), Chicago, IL 60612, USA.

Received: 21 December 2015 Accepted: 13 May 2016

Published online: 20 May 2016

\section{References}

1. Gibney SM, Drexhage HA. Evidence for a dysregulated immune system in the etiology of psychiatric disorders. J Neuroimmune Pharmacol. 2013;8(4):900-20.

2. Yolken RH, Torrey EF. Viruses, schizophrenia, and bipolar disorder. Clin Microbiol Rev. 1995;8(1):131-45

3. Kendell RE, Kemp IW. Maternal influenza in the etiology of schizophrenia. Arch Gen Psychiatry. 1989;46(10):878-82.

4. Upthegrove R, Manzanares-Teson N, Barnes NM. Cytokine function in medication-naive first episode psychosis: a systematic review and metaanalysis. Schizophr Res. 2014;155(1-3):101-8.

5. Potvin S, Stip E, Sepehry AA, Gendron A, Bah R, Kouassi E. Inflammatory cytokine alterations in schizophrenia: a systematic quantitative review. Biol Psychiatry. 2008;63(8):801-8.

6. Kubistova A, Horacek J, Novak T. Increased interleukin-6 and tumor necrosis factor alpha in first episode schizophrenia patients versus healthy controls. Psychiatr Danub. 2012;24 Suppl 1:S153-6.

7. Cole SW, Arevalo JM, Takahashi R, Sloan EK, Lutgendorf SK, Sood AK, Sheridan JF, Seeman TE. Computational identification of gene-social environment interaction at the human IL6 locus. Proc Natl Acad Sci U S A. 2010;107(12):5681-6

8. Paul-Samojedny M, Owczarek A, Kowalczyk M, Suchanek R, Palacz M, Kucia K, Fila-Danilow A, Borkowska P, Kowalski J. Association of interleukin 2 (IL-2), interleukin 6 (IL-6), and TNF-alpha (TNFalpha) gene polymorphisms with paranoid schizophrenia in a Polish population. J Neuropsychiatry Clin Neurosci. 2013;25(1):72-82

9. Zakharyan R, Petrek M, Arakelyan A, Mrazek F, Atshemyan S, Boyajyan A. Interleukin-6 promoter polymorphism and plasma levels in patients with schizophrenia. Tissue Antigens. 2012:80(2):136-42.

10. Aaltonen R, Heikkinen T, Hakala K, Laine K, Alanen A. Transfer of proinflammatory cytokines across term placenta. Obstet Gynecol. 2005; 106(4):802-7.

11. Hsiao EY, Patterson PH. Activation of the maternal immune system induces endocrine changes in the placenta via IL-6. Brain Behav Immun. 2011;25(4):604-15

12. Smith SE, Li J, Garbett K, Mirnics K, Patterson PH. Maternal immune activation alters fetal brain development through interleukin-6. J Neurosci. 2007:27(40):10695-702

13. O'Rourke RW, Kay T, Lyle EA, Traxler SA, Deveney CW, Jobe BA, Roberts CT,Jr, Marks D, Rosenbaum JT. Alterations in peripheral blood lymphocyte cytokine expression in obesity. Clin Exp Immunol. 2006;146(1):39-46. 
14. Danivas V, Venkatasubramanian G. Current perspectives on chlorpromazine equivalents: Comparing apples and oranges! Indian J Psychiatry. 2013;55(2):207-8.

15. Gardner DM, Murphy AL, O'Donnell H, Centorrino F, Baldessarini RJ. International consensus study of antipsychotic dosing. Am J Psychiatry. 2010;167(6):686-93.

16. Kay SR, Fiszbein A, Opler LA. The positive and negative syndrome scale (PANSS) for schizophrenia. Schizophr Bull. 1987;13(2):261-76.

17. Jayaraman S, Castro M, O'Sullivan M, Bragdon MJ, Holtzman MJ. Resistance to Fas-mediated T cell apoptosis in asthma. J Immunol. 1999;162(3):1717-22.

18. Mannhalter C, Koizar D, Mitterbauer G. Evaluation of RNA isolation methods and reference genes for RT-PCR analyses of rare target RNA. Clin Chem Lab Med. 2000;38(2):171-7.

19. Vandesompele J, De Preter K, Pattyn F, Poppe B, Van Roy N, De Paepe A, Speleman F (2002) Accurate normalization of real-time quantitative RT-PCR data by geometric averaging of multiple internal control genes. Genome Biol 3(7):RESEARCH0034.

20. Chase KA, Gavin DP, Guidotti A, Sharma RP. Histone methylation at H3K9: evidence for a restrictive epigenome in schizophrenia. Schizophr Res. 2013;149(1-3):15-20.

21. Pasieka TJ, Woolson RF, Grose C. Viral induced fusion and syncytium formation: measurement by the Kolmogorov-Smirnov statistical test. J Virol Methods. 2003:111(2):157-61.

22. Naudin J, Mege JL, Azorin JM, Dassa D. Elevated circulating levels of IL-6 in schizophrenia. Schizophr Res. 1996;20(3):269-73.

23. Frydecka D, Misiak B, Pawlak-Adamska E, Karabon L, Tomkiewicz A, Sedlaczek P, Kiejna A, Beszlej JA. Interleukin-6: the missing element of the neurocognitive deterioration in schizophrenia? The focus on genetic underpinnings, cognitive impairment and clinical manifestation. Eur Arch Psychiatry Clin Neurosci. 2014;265(6):449-59.

24. Karstoft K, Pedersen BK: Skeletal muscle as a gene regulatory endocrine organ. Curr Opin Clin Nutr Metab Care 2016.

25. Ikeda S, Tamura Y, Kakehi S, Sanada H, Kawamori R, Watada H. Exerciseinduced increase in IL-6 level enhances GLUT4 expression and insulin sensitivity in mouse skeletal muscle. Biochem Biophys Res Commun. 2016:473(4):947-52.

26. O'Connell KE, Thakore J, Dev KK. Pro-inflammatory cytokine levels are raised in female schizophrenia patients treated with clozapine. Schizophr Res. 2014;156(1):1-8

27. Hori H, Yoshimura R, Yamada Y, Ikenouchi A, Mitoma M, Ida Y, Nakamura J. Effects of olanzapine on plasma levels of catecholamine metabolites, cytokines, and brain-derived neurotrophic factor in schizophrenic patients. Int Clin Psychopharmacol. 2007;22(1):21-7.

28. Mengozzi M, Fantuzzi G, Faggioni R, Marchant A, Goldman M, Orencole S, Clark BD, Sironi M, Benigni F, Ghezzi P. Chlorpromazine specifically inhibits peripheral and brain TNF production, and up-regulates IL-10 production, in mice. Immunology. 1994;82(2):207-10.

29. Dimitrov DH, Lee S, Yantis J, Valdez C, Paredes RM, Braida N, Velligan D, Walss-Bass C. Differential correlations between inflammatory cytokines and psychopathology in veterans with schizophrenia: potential role for IL-17 pathway. Schizophr Res. 2013;151(1-3):29-35.

30. Frommberger UH, Bauer J, Haselbauer P, Fraulin A, Riemann D, Berger M. Interleukin-6-(IL-6) plasma levels in depression and schizophrenia: comparison between the acute state and after remission. Eur Arch Psychiatry Clin Neurosci. 1997;247(4):228-33.

31. Ganguli R, Yang Z, Shurin G, Chengappa KN, Brar JS, Gubbi AV, Rabin BS. Serum interleukin-6 concentration in schizophrenia: elevation associated with duration of illness. Psychiatry Res. 1994;51(1):1-10.

32. Haas GL, Sweeney JA. Premorbid and onset features of first-episode schizophrenia. Schizophr Bull. 1992;18(3):373-86.

33. Hoff AL, Harris D, Faustman WO, Beal M, DeVilliers D, Mone RD, Moses JA, Csernansky JG. A neuropsychological study of early onset schizophrenia. Schizophr Res. 1996;20(1-2):21-8.

34. DeLisi LE. The significance of age of onset for schizophrenia. Schizophr Bull. 1992;18(2):209-15.

35. Miller BJ, Buckley P, Seabolt W, Mellor A, Kirkpatrick B. Meta-analysis of cytokine alterations in schizophrenia: clinical status and antipsychotic effects. Biol Psychiatry. 2011;70(7):663-71.

36. Garcia de Guadiana-Romualdo L, Espanol-Morales I, Cerezuela-Fuentes P, Consuegra-Sanchez L, Hernando-Holgado A, Esteban-Torrella P, JimenezSantos E, Viqueira-Gonzalez M, de Bejar-Almira A, Albaladejo-Oton MD.
Value of lipopolysaccharide binding protein as diagnostic marker of infection in adult cancer patients with febrile neutropenia: comparison with C-reactive protein, procalcitonin, and interleukin 6. Support Care Cancer. 2015:23(7):2175-82.

37. Sun XY, Lu J, Zhang L, Song HT, Zhao L, Fan HM, Zhong AF, Niu W, Guo ZM, Dai YH, Chen C, Ding YF, Zhang LY. Aberrant microRNA expression in peripheral plasma and mononuclear cells as specific blood-based biomarkers in schizophrenia patients. J Clin Neurosci. 2015;22(3):570-4.

38. Lai CY, Yu SL, Hsieh MH, Chen CH, Chen HY, Wen CC, Huang YH, Hsiao PC, Hsiao CK, Liu CM, Yang PC, Hwu HG, Chen WJ. MicroRNA expression aberration as potential peripheral blood biomarkers for schizophrenia. PLoS One. 2011;6(6):e21635

39. Stojanovic A, Martorell L, Montalvo I, Ortega L, Monseny R, Vilella E, Labad J. Increased serum interleukin-6 levels in early stages of psychosis: associations with at-risk mental states and the severity of psychotic symptoms. Psychoneuroendocrinology. 2014;41:23-32.

40. Guest FL, Guest PC, Martins-de-Souza D. The emergence of point-of-care blood-based biomarker testing for psychiatric disorders: enabling personalized medicine. Biomark Med. 2016;10(4):431-43.

\section{Submit your next manuscript to BioMed Central and we will help you at every step:}

- We accept pre-submission inquiries

- Our selector tool helps you to find the most relevant journal

- We provide round the clock customer support

- Convenient online submission

- Thorough peer review

- Inclusion in PubMed and all major indexing services

- Maximum visibility for your research

Submit your manuscript at www.biomedcentral.com/submit
) Biomed Central 